\title{
The IL-6/JAK/STAT3 pathway: Potential therapeutic strategies in treating colorectal cancer (Review)
}

\author{
SHU-WEI WANG and YUE-MING SUN \\ Department of Minimally Invasive Surgery, The First Affiliated Hospital \\ of Nanjing Medical University, Nanjing 210029, P.R. China
}

Received December 5, 2013; Accepted January 7, 2014

DOI: $10.3892 /$ ijo.2014.2259

\begin{abstract}
Among the cytokines linked to inflammationassociated cancer, interleukin (IL)-6 drives many of the cancer 'hallmarks' through downstream activation of the Janus kinase/signal transducer and activator of transcription 3 (JAK/ STAT3) signaling pathway. Additionally, dysregulation of the interleukin (IL)-6-mediated JAK/STAT3 signaling pathway is closely related to the development of diverse human solid tumors including colorectal cancer (CRC). On this basis, modulation of the IL-6/JAK/STAT3 signaling pathway is currently being widely explored to develop novel therapies for $\mathrm{CRC}$. The present review details the mechanisms and roles of the IL-6/JAK/STAT3 pathway in CRC, describes current therapeutic strategies, and the search for potential therapeutic approaches to treat CRC.
\end{abstract}

\section{Contents}

1. Introduction

2. IL-6/JAK/STAT3 pathway

Correspondence to: Professor Yue-Ming Sun, Department of Minimally Invasive Surgery, The First Affiliated Hospital of Nanjing Medical University, 300 Guangzhou Road, Nanjing 210029, P.R. China

E-mail: sym890207@163.com

Abbreviations: IL, interleukin; IL-6R, IL-6 receptor; JAK, Janus-activated kinase; STAT3, signal transducer and activator of transcription 3; CRC, colorectal cancer; ADAM, A dsintegrin and A metalloproteinase; VEGF, vascular endothelial growth factor; TGF- $\beta$, transforming growth factor- $\beta$; Treg, regulatory $\mathrm{T}$; Th, $\mathrm{T}$ helper; MDSCs, myeloid derived suppressor cells; DCs, dendritic cells; CAC, colitis-associated cancer; IBD, inflammatory bowel disease; CD, Crohn's disease; JH, JAK homology; FERM, four-point-one, ezrin, radixin and moesin; SH2, Src homology 2; MMP, matrix metalloproteinase; McAb, monoclonal antibody; SOCS, suppressor of cytokine signaling; CIS, cytokine-inducible SH2-containing; PIAS1, Gu/RNA helicase II-binding protein; GBE, Ginkgo biloba extract; ESHS1, enoyl-CoA hydratase short chain 1

Key words: interleukin-6, Janus kinase, signal transducer and activator of transcription 3 , therapies, colorectal cancer
3. Roles of IL-6/JAK/STAT3 pathway in CRC

4. Modulation of IL-6/JAK/STAT3 pathway in CRC

5. Further perspectives

6. Conclusions

\section{Introduction}

Janus kinase (JAK)/signal transducer and activator of transcription 3 (STAT3) signaling pathway is involved in various physiological processes, including immune function, cell growth, differentiation and hematopoiesis (1). Accumulating evidence indicates that abnormalities in the JAK/STAT3 pathway play a vital role in the oncogenesis of several cancers. It was reported (2) that constitutive activation of JAK2 was found in childhood $\mathrm{T}$ cell acute lymphoblastic leukemia. Constitutive activation of STAT3 is linked to cell proliferation in breast carcinoma (3) and non-small cell lung cancer (4), and also inhibits apoptosis (5). Studies have also revealed that oncogenesis can be altered by STAT3 activation (1). These published reports all demonstrate the crucial importance of the JAK/STAT3 pathway in tumorigenesis and progression.

Colorectal cancer (CRC) is the third most common cancer worldwide and it is reported that $\sim 530,000$ patients die of the disease each year (6). Although much progress has been made in treatment, outcomes remain poor as approximately half of patients receiving treatment still die of the disease $(7,8)$. Some studies have indicated that elevated interleukin-6 (IL-6)/JAK/STAT3 signaling is one of the key pathways involving in colorectum tumorigenesis, this signaling has a critical role in various aspects including initiation, development and formation in CRC. The role of IL-6 in tumorigenesis has been well-established in CRC. Increased production of IL-6 has been reported in tumor tissue itself and in the serum of patients with CRC (9). Recent studies show that cytokinedriven JAK/STAT3 pathways play an important role in the processes of signal transduction, which are associated with the hyperproliferative and invasive phenotype of CRC cells (10). Although our knowledge of oncogenesis, protooncogene identification and tumor suppressor genes involved in the tumorigenesis of CRC are growing, the biologic and molecular mechanisms in CRC are still poorly understood. Moreover, the molecular mechanisms that control CRC progression are related to the alteration of different proto- 
oncogenes, cytokines, tumor suppressor genes and their receptors (11). Notably, these abnormalities are involved in the JAK/STAT3 signal transduction pathway.

In this review, we summarize the mechanisms and roles of IL-6/JAK/STAT3 pathway in CRC and describe current therapeutic strategies to treat CRC by targeting the IL-6/JAK/ STAT3 pathway. Importantly, we discuss how to use current knowledge to find potential therapeutic approaches.

\section{IL-6/JAK/STAT3 pathway}

Molecular cloning has identified two different forms of cellular receptors of IL-6: an 80-kDa ligand-binding chain, known as IL-6R (IL-6Ra, CD126) and a 130-kDa signal-transducing chain, gp130 (IL-6Rb, CD130). In contrast to the ubiquitous expression of gp130, IL-6R shows a highly limited expression pattern and is mainly confined to hepatocytes, leukocyte subsets and megakaryocytes (12). First, IL-6 binds to the IL-6R on target cells, then the complex of IL-6 and IL-6R contacts the gp130, thereby boosting its dimerization and the subsequent activation of intracellular signaling such as STAT3 phosphorylation by JAK. This so-called classical signaling pathway is activated during early immune responses and in turn activates the expression of various acute-phase proteins such as C-reactive protein (13). We believe that classical IL-6R signaling coordinates homeostatic properties of IL-6, which may act as a cytokine with hormone-like characteristics. In addition, a soluble type of the IL-6 receptor (sIL-6R), which is produced by limited proteolysis by A disintegrin and A metalloproteinase 10 (ADAM10) or ADAM17 of the membrane-bound IL-6R and translation from an alternatively spliced mRNA, can still bind to IL-6 and the complex of IL-6 and sIL-6R interplays with gp130. This so-called IL-6 trans-signaling represents an alternative to classical IL-6 signaling and allows IL-6 to regulate a broad spectrum of target cells including neutrophils, macrophages, epithelial cells and T cells (14). In our opinion, IL-6 trans-signaling acts as a danger signal to enhance IL-6 responsiveness and drive inflammatory events.

The signal transduction of IL-6 involves the activation of JAK, then leads to the activation of transcription factor STAT3 (15). Over 40 different cytokines or growth factors can activate STAT signaling pathway. Normally STAT3 resides in the cytoplasm. STAT3 will be phosphorylated and then forms dimers with other members of the STAT family when activated by upstream signaling pathways such as JAK, epidermal growth factor receptor and IL-6R activation. The activated STAT3 complex will then translocate from the cytoplasm to the nucleus initiating transcription of STAT3 target genes including cyclin D1, Bcl-xL, c-myc, Mcl1 and vascular endothelial growth factor (VEGF) by combining with consensus DNA elements (16). STAT3 is known to play an important role in promoting tumorigenesis of diverse human cancers (17), since it is regarded as an oncogenic transcriptional factor involving in cancer cell proliferation, differentiation, invasion, inflammation and immune function (Fig. 1).

JAK family proteins. The JAK family proteins of cytoplasmic tyrosine kinases include four mammalian members, which are related to the cytoplasmic regions of signal transducing cytokine receptors. Three of them, JAK1, JAK2 and TYK2, are expressed in various tissues, yet JAK 3 is expressed only in cells of the hematopoietic system (18). The unique structure of the JAKs easily differentiates them from other members of the protein tyrosine kinase family. The most attractive feature of these proteins is the presence of two JAK homology $(\mathrm{JH})$ domains, JH1 and JH2, which have extensive homology to tyrosine kinase domains. JH1 domain at the C-terminus appears to be a functional tyrosine kinase domain, while the $\mathrm{JH} 2$ domain does not possess substantial tyrosine kinase activity (19). The JH3-JH7 regions, which form the N-terminal half of the JAKs, are related to binding to cytokine receptors. A portion of the $\mathrm{N}$-terminal region of JAKs has similar sequence with the so-called four-point-one, ezrin, radixin and moesin (FERM) domains, and JAKs have been proved to shield a divergent type of FERM domain. The SH2 domain also contains JH3 and JH4 domains. The selectivity of STAT activation by various ligands mainly depends on the highly specific interactions between the $\mathrm{SH} 2$ domain and the phosphotyrosine residues on each receptor (20) (Fig. 2).

STAT family proteins. STAT family proteins have identified seven mammalian members: STAT1, STAT2, STAT3, STAT4, STAT5 (STAT5 $\alpha$ and STAT5 $\beta$ ), and STAT6. Every STAT protein has several conserved domains including the $\mathrm{N}$-terminal coiled-coil domain, DNA binding domain, a linker, Src homology 2 (SH2) domain, and a C-terminal transactivation domain, which are closely related to its functions (21). Several studies have indicated that the N-terminal domain promotes dimerized STAT molecules to polymerize and bind multiple DNA sites that are related to oncogenic growth signaling pathways (22). The DNA-binding domain is the central region of each STAT protein (except for STAT2) and regulates the DNA-binding specificity for each STAT protein (23). A linker domain, region of 500-575, is $\alpha$-helical before the classical SH2 domain. The conserved $\mathrm{SH} 2$ domain exists in the region between 600 and 700 amino acid residues and it is crucial to form dimers between two activated STAT monomers via reciprocal phospho-tyrosine-SH2-domain interactions (20). The C-terminal transactivation domain is associated with transcriptional complexes, and builds structures when binding with interacting partners. The tyrosine residue of position 705 in this STAT domain plays an important role in STAT activation. Additionally, at position 727 (except STAT2 and STAT6), a conserved serine, is a phosphorylation site and regulates STAT transcriptional activity (24) (Fig. 2).

\section{Roles of IL-6/JAK/STAT3 pathway in CRC}

IL-6 in CRC. IL-6, a pleiotropic inflammatory cytokine, not only has central roles in immune and inflammatory response, but also is regarded as a key growth factor for malignancy (25). Cancer cells utilize the autocrine production of growth and survival factors to upregulate growth and survival pathways. The increased expression of IL-6 in cancer cells manifests that IL-6 is an important autocrine growth factor promoting tumorigenesis (26). Tissue or infection will cause more secretion of IL-6. Thereby, IL-6 participates in the regulation of the acute phase response, the differentiation of monocytes 


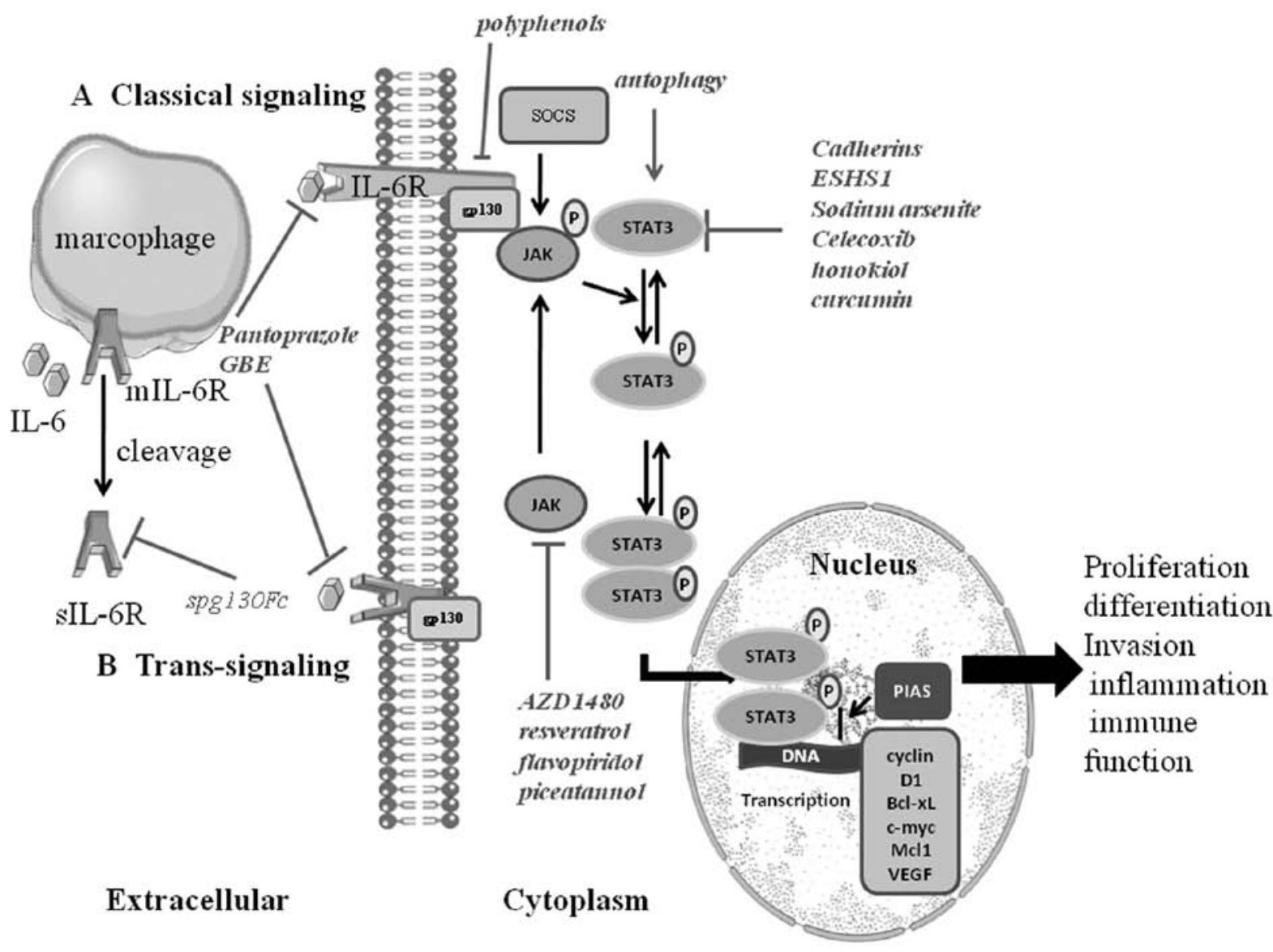

Figure 1. IL-6/JAK/STAT3 signaling pathway. IL-6 activation has two modes of: (A) classical signaling pathway: IL-6 binds to the IL-6R on target cells. Then the complex of IL-6 and IL-6R contacts the gp130; (B) trans-signaling pathway: a soluble type of the IL-6 receptor (sIL-6R) binds to IL-6 and interplays with gp130. Thereby both of them induce dimerization and initiates signaling. The signal transduction of IL-6 involves the activation of JAK, then leading to the activation of transcription factors of the signal transducers and activators of STAT3. Normally STAT3 resides in the cytoplasm. STAT3 is phosphorylated and then forms dimers with other members of the STAT family when activated by upstream signaling pathways such as JAK, EGFR, IL-6 receptor activation. The activated STAT3 complex will then translocate from the cytoplasm to the nucleus initiating transcription of STAT3 target genes (including cyclin D1, Bcl-xL, c-myc, Mcl1, survivin and VEGF) by combining with consensus DNA elements. STAT3 is regarded as an oncogenic transcriptional factor involved in cancer cell proliferation, differentiation, invasion, inflammation and immune function. Many factors can influence the range and duration of STAT activation. The protein inhibitors of activated STATs (PIAS) family of proteins are negative regulators of STAT-mediated gene transcription. In addition, the suppressors of cytokine signaling (SOCS) protein family affects the JAKs, and thus restrain the phosphorylation of gp130, STATs and the JAKs themselves.

A Structure of JAK

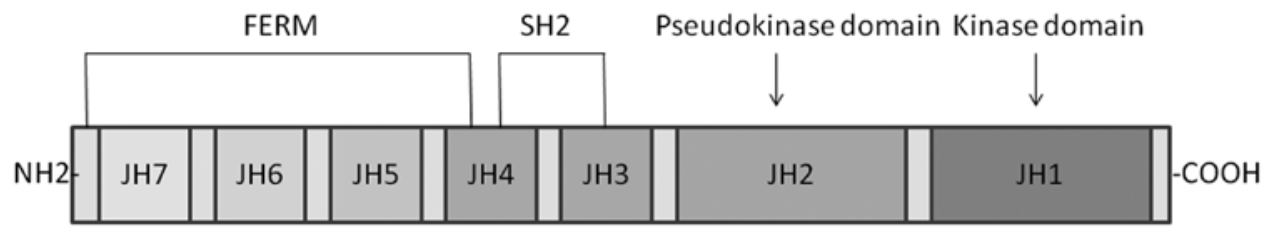

B Structure of STAT3 isoforms

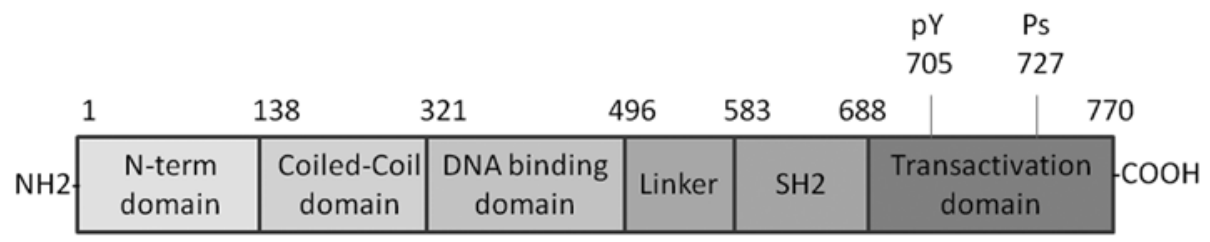

Figure 2. Structure of JAK and the STAT3 isoforms. (A) The structural domains of JAK are referred to as JAK homology regions (JH1-JH7). JAK also possesses four functional domains: the FERM domain, the SH2 domain, the pseudotyrosine kinase (TK) domain and a catalytically active TK domain. (B) Structure of the STAT3 isforms including the N-terminal coiled-coil domain, DNA binding domain, a linker, Src homology 2 (SH2) domain, and a C-terminal transactivation domain are shown, as well as the tyr705 and ser727 phosphorylation sites. 
to macrophages, the proliferation and apoptotic resistance of T cells and Th 2 cytokine production (27). The IL-6 signaling pathway is considered as one of the most important ways linking inflammation to cancer (28).

The role of IL-6 in tumorigenesis has been well-established in a wide range of human cancers including lymphoma, glioma, melanoma, breast, ovarian, prostate, renal, pancreatic cancer, as well as CRC. An increasing number of studies have found that IL-6 levels elevate in tumor tissue itself and in the serum of patients with CRC (9). Additionally, a review survey by Knüpfer and Preiss shows that IL-6 expression is closely related to tumor stage, size, metastasis and survival of patients with CRC (29). IL-6/sIL-6R and inflammation function in the pathogenesis of CRC (30). IL-6 plays an important role in recruitment of immune cells that produce pro-inflammatory cytokines, and also in modulating Th17 and Treg cells in CRC (31). Moreover, IL-6 was reported to be localized at the sites of macrophage infiltration, suggesting an interaction between IL- 6 and immune cells in the tumor microenvironment (32). Two recognized IL-6 polymorphisms have been reported to associate with a significantly reduced risk of CRC. In a study of 46 patients with $\mathrm{CRC}$, multivariate analysis showed that the blood granulocyte/lymphocyte ratio and the serum IL-6 level were independent risk factors for poor prognosis, indicating that both factors may be significantly predictive for CRC cancer progression (33).

The risk of developing precancerous lesions and invasive carcinoma will increase exponentially when under the duration of inflammation in inflammatory bowel disease (IBD) patients such as Crohn's disease (CD) and ulcerative colitis (UC), and those who have not controlled inflammation will have higher risk of development of CAC (27). There is a $17.8 \%$ risk for CRC in patients with UC within 30 years (34). The cumulative risk for the development of $\mathrm{CRC}$ in patients with large bowel involvement of $\mathrm{CD}$ is $\sim 8.3 \%$ over a period of 30 years (35). Similar to CRC, IL-6 expression is increased in patients with IBD (36). Many studies have manifested that IL-6 plays central roles in the pathogenesis of IBD, mainly due to its effect on immune cell function (27). IL-6 trans-signaling has been proved to activate $\mathrm{T}$ cells in the lamina propria of patients with IBD and leads to resistance of these cells against apoptosis via upregulation of anti-apoptotic factors such as Bcl-2 and Bcl-xL (37). According to the association of IL-6 expression with $\mathrm{CRC}$ prognosis and the increased expression of IL-6 in patients with IBD, IL-6 is known as a connection between chronic inflammation and tumor development. New data revealed a direct effect of a functional relevance for IL-6 acting on tumor cells in sporadic CRC, which is likely mediated by trans-signaling, as intestinal epithelial cells usually do not express mIL-6R (38).

JAK/STAT3 in CRC. Similarly to many malignant tumors, the hyperproliferative and invasive phenotype of CRC cells has showed to be related to abnormalities on the level of signal transduction. Cytokine-driven JAK/STAT3 pathways play crucial roles in these processes (10). Generally, the molecular mechanisms that regulate development of CRC are associated with the expression of different proto-oncogenes, tumor suppressor genes, cytokines, and their receptors, including Ras, Src, p27 ${ }^{\mathrm{kip} 1}, \mathrm{p} 16^{\mathrm{ink4a}}$, interleukin and epidermal growth factor receptor. These alterations markedly refer to the JAK/ STAT3 signal transduction pathway (39). Though few studies of abnormal expression of JAK/STAT3 have been shown in CRC, STAT3 activity is constitutively upregulated in diverse human tumors including CRC (11).

So far several studies have indicated that elevated malignancy and invasive behaviour of CRC cells are closely associated with STAT3 activity (40). STAT3 is originally characterized as a mediator of IL- 6 receptor signaling (41) with extremely widespread functions throughout the organism (reviewed in ref. 42) and it is the only embryonic lethal knockout within the STAT family (43). Not only cytokine and growth factor receptors, but several viral or cellular oncogenes such as src, fps, polyoma virus middle T-antigen, and sis are also known to activate STAT3 (reviewed in ref. 44). A constitutively active artificial variant of STAT3 generated by forced dimerization was shown to behave as an oncoprotein to induce tumorigenesis in nude mice (45). STAT3 activity is thought linked to elevated malignancy and invasive behaviour of CRC cells. STAT3 activation can increase the expression of matrix metalloproteinase (MMP), which also potentially promote CRC cells invasion and metastasis via proteolytic degradation of the extracellular matrix (46). The first evidence has be provided that Aloin may inhibit tumor angiogenesis and growth by inhibiting STAT3 activation (47). Also recently it has been demonstrated that STAT3 signaling is important for the development of CRC and promotes angiogenesis by regulating VEGF-A and MMP2 expression (48). However, some research showed that STAT3 activity may also have negative effects on the development of colon cancer. From Apc ${ }^{\mathrm{Min}}$ mouse models, STAT3 was demonstrated to inhibit tumor cell invasiveness and adenoma to carcinoma transition (49).

\section{Modulation of IL-6/JAK/STAT3 pathway in CRC}

Although in recent years significant progress was made in $\mathrm{CRC}$ treatment, $\mathrm{CRC}$ still remains the leading cause of cancer related to death in the worldwide. Therefore, new therapeutic methods, especially for patients with advanced disease, are greatly required. Therapeutics targeting the IL-6/JAK/ STAT3 pathway are hopeful strategies because more and more evidence shows that IL-6/JAK/STAT3 pathway has a critical role in various aspects including initiation, development and formation in CRC.

Regulation of IL-6. IL-6 is an important tumor promoting cytokine that enforces proliferation and anti-apoptotic effects in tumor cells. Clinical and experimental data strongly propose a contribution of IL-6 signaling to the development of both sporadic and colitis-associated CRC development. In this regard, several components of the IL- 6 signaling pathway such as IL-6R have been proposed as promising targets for CRC therapy.

Several studies have reported that IL-6 and STAT3 play an important role in the survival of intestinal epithelial cells and development of inflammation-associated cancer, anti-IL6R antibody (anti-IL-6RAb) has been reported as an inhibitor to suppress CRC development (50). Subsequently, IL-6 ligand-blocking antibody was produced to express functions 
Table I. Current therapies targeting the IL-6/JAK/STAT3 pathway in CRC.

\begin{tabular}{llll}
\hline Strategy & \multicolumn{1}{c}{ Compound } & \multicolumn{1}{c}{ Disease } & Phase \\
\hline IL-6 inhibition & CNTO328 & Solid cancer including CRC & Phase I + II \\
& Oroxylin A & Experimental model of cancer & Preclinical \\
IL-6R inhibition and & Anti-IL-6R antibody & Experimental model of cancer & Preclinical \\
SIL-6R inhibition & (tocilizumab) & CRC & Clinical \\
Stat3 inhibition & Hedyotis diffusa Willd, Spica Prunellae & & Preclinical \\
\hline JAK/STAT signaling inhibition & Trichostatin A, bufalin & Experimental CRC & \\
\hline
\end{tabular}

in antitumor and anti-inflammatory activities. CNTO-328, a human-mouse chimeric antibody, was generated from a murine anti IL-6 monoclonal antibody (McAb). CNTO-328 has a long half-life ( 2 weeks) without remarkable immunogenicity and it also has a strong affinity with recombinant as well as native IL-6 (51). It is capable of blocking the signal transduction pathway of IL-6/IL-6R/gp130 by inhibiting the binding of IL-6 to the IL-6R, ultimately achieving antitumor and anti-inflammatory effects (52). In addition, the humanized anti-IL-6R McAb of IgG1 class, named anti-IL-6R Ab (tocilizumab), was produced by grafting the complementarity-determining regions of a mouse antihuman IL-6R antibody onto human IgG1. The mechanism of anti-IL-6R Ab is to control IL-6-mediated signal transduction by inhibiting the process of IL- 6 binding to membrane-bound IL-6R and sIL-6R (53). Tocilizumab was reported to treat Castelman's disease and rheumatoid arthritis and may be used in cancers (54). Recently, it was shown that when expression of IL-6 and IL- $1 \beta$ decreases, the IL-6/STAT3 signaling pathway also weakens in tumors in oroxylin A-treated mice. Through this mechanism it was demonstrated that oroxylin A inhibits colitis-associated carcinogenesis in an azoxymethane/ dextran sodium sulfate mouse model and in the colon cancer cell line HCT-116 (55) (Table I).

The above treatments work in both classical and transsignaling, and therefore also block physiological functions of IL-6. In contrast, modulation of sgp130Fc is a specific inhibition of trans-signaling. $\mathrm{Sgp} 130 \mathrm{Fc}$ is a designer cytokine that specifically binds IL-6/sIL-6R complexes, and therefore it only blocks trans-signaling. It has been shown to be effective for the treatment of experimental CAC with sgp130Fc in the study by Becker et al (56). The substance will soon enter clinical development and it will be interesting to evaluate its effect on human cancer (57).

Regulation of JAK/STAT3. JAK/STAT3 has been shown in many aspects of CRC development, including cell growth, survival, invasion and migration $(39,58)$. Therefore, inhibiting JAK/STAT3 is a valuable regulative strategy for cancers.

The suppressor of cytokine signaling (SOCS) proteins family comprise of eight members: SOCS 1-7, and cytokineinducible SH2-containing (CIS)-1 (with similar structure to the other SOCS proteins). So far research has mainly focused on CIS and SOCS1-3, which have been found to serve as nega- tive regulators of the JAK/STAT signaling pathway. However, the various family members seem to have different specific mechanism. CIS, first defined member, was proved to interact with STAT5 and then regulate the JAK2/STAT5 pathway. SOCS1 and SOCS3 are the most effective in inhibiting IL-6mediated signaling pathways. Evidence has shown that both of above members affect the JAKs, thus restraining the phosphorylation of gp130, STATs and the JAKs themselves (59). Some studies reported that SOCS3 expression was decreased in a variety of inflammation-associated human cancers and cancer cell lines, which was correlated with strong STAT3 activity in these cells (60). New data demonstrate that sodium butyrate inhibits JAK2/STAT signaling through upregulation of SOCS1 and SOCS3, which are regulated by histone deacetylase $8(61)$. Likewise, a study shows that honokiol increases the activity and protein expression of SH2-containing tyrosine phosphatase-1 further blocking the STAT3 pathway (62) (Fig. 2). It has been demonstrated that, like in various other tumors with high STAT3 activity, DNA methylation influences epigenetic regulation leading to the decline in SOCS3 expression in CRC (32). In addition, data exist suggesting that trichostatin A may increase SOCS1 and SOCS3 expression by inducing histone modifications and ultimately inhibit JAK2/STAT3 signaling in CRC cells (63) (Table I).

The protein inhibitors of activated STATs (PIAS) family of proteins are negative regulators of STAT-mediated gene transcription. The family of PIAS consists of five mammalian components: PIAS1, PIAS3, PIASx $\alpha$, PIASx $\beta$ and PIASy. Since Gu/RNA helicase II-binding protein (PIAS1) was recognized, it is widely accepted that PIASs are transcriptional co-regulator proteins important to the JAK/STAT pathway (64). Inhibitory molecules from PIAS can interact with activated STATs, but they inhibit different STAT proteins by distinct types. For instance, PIAS3 inhibits STAT3-mediated gene expression (after IL-6 stimulation), whereas PIAS1 blocks STAT1-dependent signaling and directly inhibits the STAT-DNA complex activity with other transcriptional suppressive co-operators (65). Recent studies have shown that curcumin controls activation of PIAS-3 to restrain JAK-STAT signaling, thus weakens STAT-3 phosphorylation and tumor cell growth (66) (Fig. 1). Inhibitor of activated STAT3 protein-PIAS3 expression was also reduced in various cancers including prostate, gastric, brain, and CRC (67). 
Table II. Potential therapies targeting of the IL-6/JAK/STAT3 pathway in CRC.

\begin{tabular}{lll}
\hline Strategy & \multicolumn{1}{c}{ Compound } & \multicolumn{1}{c}{ Indication } \\
\hline IL-6 inhibition & Pantoprazole & Gastric cancer \\
& GBE & Endothelial inflammation \\
IL-6R inhibition and SIL-6R & Polyphenols & Suppress angiogenesis \\
inhibition & REGN-88 & Rheumatoid arthritis \\
JAK inhibition & AZD1480 & Solid tumors \\
& Resveratrol flavopiridol piceatannol & Hematologic malignancies \\
& CEP-701 & Reduce cytokine production \\
& XL019 & \\
INCB018424 & Myelofibrosis \\
& & Myeloproliferative disorders \\
Cadherins & Hematologic malignancies \\
& ESHS1 & Control survival \\
& Sodium arsenite & \\
& Celecoxib & Induce apoptosis \\
& Honokiol & Induce cell apoptosis on \\
& Curcumin & nasopharyngeal carcinoma \\
& STAT3 decoy & \\
\hline
\end{tabular}

Novel agents directly inhibiting STAT3 were designed mainly to target the SH2 domain, which block either STAT3 phosphorylation or dimerization. These contain designed small molecules and peptidomimetics. Studies from many preclinical cancer models, many of these agents with high specificity to disrupt STAT3 function have been found to inhibit cancer growth (68). However, these agents have not been applied in clinic (69). Continuous unregulated STAT3 could increase cell proliferation and reduce cell apoptosis, then leading to development of various cancers including CRC (39,70). Therefore, affecting the STAT3 pathway and its target gene expression could balance cell apoptosis with proliferation, which seems a promising strategy for the development of novel anticancer therapies. Recent studies have showed that treatment with some well-known traditional Chinese formulas such as Pien Tze Huang (71), ethanol extract of Hedyotis diffusa Willd (72), Spica Prunellae (73) would lead to the inhibition of cancer cell proliferation and the promotion of apoptosis in CRC mouse tumor tissues through suppression of STAT3 phosphorylation activation (Table I).

Since the IL-6/JAK/STAT3 pathway is important to CRC, research has been conducted to reveal its potential role in treating CRC. Abrogation of galectin- 4 expression promotes cancer cell proliferation and the downregulation of galectin-4 elicits tumor promotion in vitro and in vivo through activation of IL-6/NF- $x$ B/STAT3 signaling, so regulation of galectin- 4 may be a direction to treat CRC (74). Organo-Mg inhibits inflammation-related mouse colon carcinogenesis by modulating the proliferative activities and chromosomal instability of CRC and suppressing colonic inflammation suggests potential use of organo- $\mathrm{Mg}$ for clinical chemoprevention trials of CRC in the inflamed colon (75). Leptin influences the growth and proliferation of cancer cells via activation of various growth and survival signaling pathways including JAK/STAT, PI3-kinase/AKT, and/or MAP kinases, showing promise as a molecule to treat CRC (76). Ganetespib, a potent heat shock protein 90 (HSP90) inhibitor disrupts angiogenesis in CRC through inhibition of HIF-1 $\alpha$ and STAT-3 CRC cell lines (HCT116 and HT29), and these results collectively suggest that inhibition of HSP90 is a promising anti-angiogenic strategy in CRC (77). Recently studies have showed that bufalin not only inhibits the growth of CRC SW620 cells, but also induces apoptosis of SW620 cells through inhibition of JAK/STAT3 signaling pathway (78), thus, more clinical studies are required to confirm the efficacy of bufalin to treat human CRC.

\section{Further perspectives}

To date, many studies have recorded potential therapies in IL-6/JAK/STAT3 pathway to treat many cancers. However, limited attention has been paid to CRC, even though IL-6/ JAK/STAT3 pathway plays a vital role in CRC. The studies in other diseases have indicated the influence on relieving the pathology in IL-6/JAK/STAT3 pathway, and it indicates a promising future in CRC, while more studies need to be performed to verify the hypothesis.

Some studies have demonstrated that modulating the expression of IL- 6 can prevent the development of other diseases. Pantoprazole decreased the secretion of IL-6 and 
caused cell death specifically in gastric cancer. Therefore, it may be a potential substance to treat CRC, which shows similar mechanism of tumor progression to gastric cancer (79). Dietderived polyphenols suppressed angiogenesis by regulating the expression of IL-6 signal transducing receptor (IL-6R $\alpha$ ) and SOCS3 protein, which is also the signaling pathway inhibiting development of CRC (80). Ginkgo biloba extract (GBE) inhibited high-glucose-induced endothelial inflammation by restraining redox-dependent IL-6 pathways, so GBE may be a potential target to relieve intestinal inflammation, which relates to inflammation-associated CRC (81).

Another method is to specifically inhibit JAK activation, which involves the activation of transcription factor STAT3. Some preclinical trials have utilized a number of natural products such as resveratrol, flavopiridol and piceatannol to inhibit pathways involved in inflammation, whose mechanisms include inhibition of STAT3 phosphorylation, reduction of the cytokine production and direct inhibition of the JAK (69). The role of JAK inhibition in solid tumors was also tested preclinically. The JAK1/2 inhibitor AZD1480 suppressed tumor development in models of IL-6-driven breast, ovarian, and prostate cancers (82). Currently, there is little clinical data on the use of JAK inhibition in CRC. However, AZD1480 and these natural products may be potential substances to treat $\mathrm{CRC}$ in the future.

Regulating the activation of STAT3 can prevent the progress of diseases. Three classical cadherins, E-cadherin, $\mathrm{N}$-cadherin and cadherin 11, can control survival via the gp130/STAT3 pathway, thus we may control cadherins to inhibit the progress of CRC through the STAT3 pathway (83). Enoyl-CoA hydratase short chain 1 (ESHS1) specifically inhibited STAT3 activity and decreased expression of several target genes of STAT3 (84). Additionally, sodium arsenite inhibited self-renewal and induced apoptosis in mouse embryonic stem cells, which was enhanced also by suppressing the STAT3 pathways simultaneously (85). Therefore, functions of ECHS1 and sodium arsenite may also come true in intestinal cells, which prevent the development of CRC via the STAT3 pathway. Celecoxib induced cell apoptosis and cell cycle arrest on nasopharyngeal carcinoma, which was partly mediated by the STAT3 pathway. So it may be used as the promising target to induce intestinal cell apoptosis and treat CRC, but more studies should be performed (86). Advanced glycation end product-specific receptor-mediated autophagy contributed to pancreatic tumorigenesis and bioenergetics via the IL6-pSTAT3 pathway, so inhibiting the receptor could be a potential therapeutic manner to control tumorigenesis of CRC and other solid tumors (87) (Fig. 1 and Table II).

Some studies show that combining with inhibition of the IL-6/STAT3 signaling can enhance the effect of chemoradiotherapy. Treatment together with IL-6 inhibition enhanced the radiation response of prostate cancer (88). Ganoderic acid A, inhibition of the JAK-STAT3 signaling pathway, increased chemosensitivity of HepG2 cells to cisplatin (89). Therefore, the inhibition may be applied to treat CRC and other solid tumors needing chemoradiotherapy.

Putoczki et al have revealed that the related cytokine IL-11 might have a stronger correlation with elevated STAT3 activation in human gastrointestinal cancers in genetic mouse models (90). Therefore, targeting the interference with IL-11 could be a potential therapeutic strategy for the treatment of gastrointestinal cancers.

\section{Conclusions}

The continuous evidence of recent years makes us convinced that IL-6/JAK/STAT3 pathway plays a crucial role in colorectum tumorigenesis. The use of inhibitors of this signal transduction pathway has provided critical information for a better understanding of molecular mechanisms of pathology and developing new therapeutic methods of CRC. Thus, strategies targeting the IL-6/JAK/STAT3 pathway have emerged as attractive options to treat CRC.

Many studies have showed the potential therapy of IL-6/ STAT3 signaling pathway to various diseases. However, experimental research on the treatment of CRC in IL-6/JAK/ STAT3 signaling pathway is limited, even if this signaling is one of the key pathways involved in colorectum tumorigenesis. The studies in other diseases have demonstrated the influence on relieving the pathology in IL-6/JAK/STAT3 pathway, and it indicates a promising future in CRC, while more research needs to be carried out to confirm the hypothesis.

\section{References}

1. Niwa Y, Kanda H, Shikauchi Y, et al: Methylation silencing of SOCS-3 promotes cell growth and migration by enhancing JAK/ STAT and FAK signalings in human hepatocellular carcinoma. Oncogene 24: 6406-6417, 2005.

2. Lacronique V, Boureux A, Valle VD, et al: A TEL-JAK2 fusion protein with constitutive kinase activity in human leukemia. Science 278: 1309-1312, 1997.

3. Zhang F, Li C, Halfter $\mathrm{H}$ and Liu J: Delineating an oncostatin M-activated STAT3 signaling pathway that coordinates the expression of genes involved in cell cycle regulation and extracellular matrix deposition of MCF-7 cells. Oncogene 22: 894-905, 2003.

4. Alvarez JV, Greulich H, Sellers WR, Meyerson M and Frank DA: Signal transducer and activator of transcription 3 is required for the oncogenic effects of non-small-cell lung cancer-associated mutations of the epidermal growth factor receptor. Cancer Res 66: 3162-3168, 2006.

5. Zamo A, Chiarle R, Piva R, et al: Anaplastic lymphoma kinase (ALK) activates Stat 3 and protects hematopoietic cells from cell death. Oncogene 21: 1038-1047, 2002.

6. Khong TL, Thairu N, Larsen H, Dawson PM, Kiriakidis S and Paleolog EM: Identification of the angiogenic gene signature induced by EGF and hypoxia in colorectal cancer. BMC Cancer 13: 518,2013

7. Guthrie GJ, Roxburgh CS, Horgan PG and McMillan DC: Does interleukin-6 link explain the link between tumour necrosis, local and systemic inflammatory responses and outcome in patients with colorectal cancer? Cancer Treat Rev 39: 89-96, 2013.

8. Siegel R, Naishadham D and Jemal A: Cancer statistics, 2012. CA Cancer J Clin 62: 10-29, 2012.

9. Chung YC and Chang YF: Serum interleukin-6 levels reflect the disease status of colorectal cancer. J Surg Oncol 83: 222-226, 2003.

10. Gordziel C, Bratsch J, Moriggl R, Knosel T and Friedrich K: Both STAT1 and STAT3 are favourable prognostic determinants in colorectal carcinoma. Br J Cancer 109: 138-146, 2013.

11. Dambacher J, Beigel F, Seiderer J, et al: Interleukin 31 mediates MAP kinase and STAT1/3 activation in intestinal epithelial cells and its expression is upregulated in inflammatory bowel disease. Gut 56: 1257-1265, 2007.

12. Kishimoto T: IL-6: from its discovery to clinical applications. Int Immunol 22: 347-352, 2010.

13. Rose-John S: Coordination of interleukin- 6 biology by membrane bound and soluble receptors. Adv Exp Med Biol 495: 145-151, 2001. 
14. Culig Z: Cytokine disbalance in common human cancers Biochim Biophys Acta 1813: 308-314, 2011.

15. Heinrich PC, Behrmann I, Haan S, Hermanns HM, MullerNewen G and Schaper F: Principles of interleukin (IL)-6-type cytokine signalling and its regulation. Biochem J 374: 1-20, 2003.

16. Aoki Y, Feldman GM and Tosato G: Inhibition of STAT3 signaling induces apoptosis and decreases survivin expression in primary effusion lymphoma. Blood 101: 1535-1542, 2003.

17. Leeman RJ, Lui VW and Grandis JR: STAT3 as a therapeutic target in head and neck cancer. Expert Opin Biol Ther 6: 231-241, 2006.

18. Haan C, Kreis S, Margue C and Behrmann I: Jaks and cytokine receptors - an intimate relationship. Biochem Pharmacol 72: 1538-1546, 2006.

19. Rane SG and Reddy EP: JAKs, STATs and Src kinases in hematopoiesis. Oncogene 21: 3334-3358, 2002.

20. Subramaniam A, Shanmugam MK, Perumal E, et al: Potential role of signal transducer and activator of transcription (STAT)3 signaling pathway in inflammation, survival, proliferation and invasion of hepatocellular carcinoma. Biochim Biophys Acta 1835: 46-60, 2013.

21. Fan Y, Mao R and Yang J: NF-kappaB and STAT3 signaling pathways collaboratively link inflammation to cancer. Protein Cell 4: 176-185, 2013.

22. Xu Q, Briggs J, Park S, et al: Targeting Stat3 blocks both HIF-1 and VEGF expression induced by multiple oncogenic growth signaling pathways. Oncogene 24: 5552-5560, 2005.

23. Ma J and Cao X: Regulation of Stat 3 nuclear import by importin alpha5 and importin alpha7 via two different functional sequence elements. Cell Signal 18: 1117-1126, 2006.

24. Shuai K: The STAT family of proteins in cytokine signaling. Prog Biophys Mol Biol 71: 405-422, 1999.

25. Candido J and Hagemann T: Cancer-related inflammation. J Clin Immunol 33 (Suppl 1): S79-S84, 2013.

26. Grivennikov S and Karin M: Autocrine IL-6 signaling: a key event in tumorigenesis? Cancer Cell 13: 7-9, 2008.

27. Neurath MF and Finotto S: IL-6 signaling in autoimmunity, chronic inflammation and inflammation-associated cancer. Cytokine Growth Factor Rev 22: 83-89, 2011.

28. Naugler WE and Karin M: The wolf in sheep's clothing: the role of interleukin-6 in immunity, inflammation and cancer. Trends Mol Med 14: 109-119, 2008.

29. Knupfer $\mathrm{H}$ and Preiss R: Serum interleukin-6 levels in colorectal cancer patients - a summary of published results. Int J Colorectal Dis 25: $135-140,2010$.

30. Atreya R and Neurath MF: Involvement of IL- 6 in the pathogenesis of inflammatory bowel disease and colon cancer. Clin Rev Allergy Immunol 28: 187-196, 2005.

31. Jones SA, Richards PJ, Scheller J and Rose-John S: IL-6 transsignaling: the in vivo consequences. J Interferon Cytokine Res 25 : 241-253, 2005.

32. Li Y, de Haar C, Chen M, et al: Disease-related expression of the IL6/STAT3/SOCS3 signalling pathway in ulcerative colitis and ulcerative colitis-related carcinogenesis. Gut 59: 227-235, 2010.

33. Shimazaki J, Goto Y, Nishida K, Tabuchi T, Motohashi G and Ubukata H: In patients with colorectal cancer, preoperative serum interleukin-6 level and granulocyte/lymphocyte ratio are clinically relevant biomarkers of long-term cancer progression. Oncology 84: 356-361, 2013.

34. Jess T, Rungoe C and Peyrin-Biroulet L: Risk of colorectal cancer in patients with ulcerative colitis: a meta-analysis of population-based cohort studies. Clin Gastroenterol Hepatol 10: 639-645, 2012.

35. Canavan C, Abrams KR and Mayberry J: Meta-analysis: colorectal and small bowel cancer risk in patients with Crohn's disease. Aliment Pharmacol Ther 23: 1097-1104, 2006.

36. Atreya R and Neurath MF: New therapeutic strategies for treatment of inflammatory bowel disease. Mucosal Immunol 1: $175-182,2008$.

37. Atreya R, Mudter J, Finotto S, et al: Blockade of interleukin 6 trans signaling suppresses $\mathrm{T}$-cell resistance against apoptosis in chronic intestinal inflammation: evidence in crohn disease and experimental colitis in vivo. Nat Med 6: 583-588, 2000.

38. Yamamoto K and Rose-John S: Therapeutic blockade of interleukin-6 in chronic inflammatory disease. Clin Pharmacol Ther 91: 574-576, 2012.
39. Xiong H, Zhang ZG, Tian XQ, et al: Inhibition of JAK1, 2/STAT3 signaling induces apoptosis, cell cycle arrest, and reduces tumor cell invasion in colorectal cancer cells. Neoplasia 10: 287-297, 2008.

40. Xiong H, Hong J, Du W, et al: Roles of STAT3 and ZEB1 proteins in E-cadherin down-regulation and human colorectal cancer epithelial-mesenchymal transition. J Biol Chem 287: 5819-5832, 2012.

41. Akira S, Nishio Y, Inoue M, et al: Molecular cloning of APRF, a novel IFN-stimulated gene factor 3 p91-related transcription factor involved in the gp130-mediated signaling pathway. Cell 77: 63-71, 1994.

42. Levy DE and Lee CK: What does Stat3 do? J Clin Invest 109: 1143-1148, 2002.

43. Takeda K, Noguchi K, Shi W, et al: Targeted disruption of the mouse Stat 3 gene leads to early embryonic lethality. Proc Natl Acad Sci USA 94: 3801-3804, 1997.

44. Bowman T, Garcia R, Turkson J and Jove R: STATs in oncogenesis. Oncogene 19: 2474-2488, 2000.

45. Bromberg JF, Wrzeszczynska MH, Devgan G, et al: Stat 3 as an oncogene. Cell 98: 295-303, 1999.

46. Zugowski C, Lieder F, Muller A, et al: STAT3 controls matrix metalloproteinase-1 expression in colon carcinoma cells by both direct and AP-1-mediated interaction with the MMP-1 promoter. Biol Chem 392: 449-459, 2011.

47. Pan Q, Pan H, Lou H, Xu Y and Tian L: Inhibition of the angiogenesis and growth of Aloin in human colorectal cancer in vitro and in vivo. Cancer Cell Int 13: 69, 2013.

48. Qian WF, Guan WX, Gao Y, et al: Inhibition of STAT3 by RNA interference suppresses angiogenesis in colorectal carcinoma. Brazilian journal of medical and biological research $=$ Revista brasileira de pesquisas medicas e biologicas / Sociedade Brasileira de Biofisica ... [et al.] 44: 1222-1230, 2011.

49. Lee J, Kim JC, Lee SE, et al: Signal transducer and activator of transcription 3 (STAT3) protein suppresses adenoma-tocarcinoma transition in Apcmin/+ mice via regulation of Snail-1 (SNAI) protein stability. J Biol Chem 287: 18182-18189, 2012.

50. Grivennikov S, Karin E, Terzic J, et al: IL-6 and Stat3 are required for survival of intestinal epithelial cells and development of colitis-associated cancer. Cancer Cell 15: 103-113, 2009

51. Guo Y, Xu F, Lu T, Duan Z and Zhang Z: Interleukin-6 signaling pathway in targeted therapy for cancer. Cancer Treat Rev 38: 904-910, 2012.

52. Puchalski T, Prabhakar U, Jiao Q, Berns B and Davis HM: Pharmacokinetic and pharmacodynamic modeling of an antiinterleukin-6 chimeric monoclonal antibody (siltuximab) in patients with metastatic renal cell carcinoma. Clin Cancer Res 16: 1652-1661, 2010.

53. Tanaka T, Narazaki M and Kishimoto T: Therapeutic targeting of the interleukin-6 receptor. Annu Rev Pharmacol Toxicol 52: 199-219, 2012.

54. Garnero P, Thompson E, Woodworth T and Smolen JS: Rapid and sustained improvement in bone and cartilage turnover markers with the anti-interleukin- 6 receptor inhibitor tocilizumab plus methotrexate in rheumatoid arthritis patients with an inadequate response to methotrexate: results from a substudy of the multicenter double-blind, placebo-controlled trial of tocilizumab in inadequate responders to methotrexate alone. Arthritis Rheum 62: 33-43, 2010.

55. Yang X, Zhang F, Wang Y, et al: Oroxylin A inhibits colitisassociated carcinogenesis through modulating the IL-6/STAT3 signaling pathway. Inflamm Bowel Dis 19: 1990-2000, 2013.

56. Becker C, Fantini MC, Schramm C, et al: TGF-beta suppresses tumor progression in colon cancer by inhibition of IL-6 transsignaling. Immunity 21: 491-501, 2004.

57. Waetzig GH and Rose-John S: Hitting a complex target: an update on interleukin-6 trans-signalling. Expert Opin Ther Targets 16: 225-236, 2012

58. Xiong H, Su WY, Liang QC, et al: Inhibition of STAT5 induces G1 cell cycle arrest and reduces tumor cell invasion in human colorectal cancer cells. Lab Invest 89: 717-725, 2009.

59. Trengove MC and Ward AC: SOCS proteins in development and disease. Am J Clin Exp Immunol 2: 1-29, 2013.

60. Isomoto $\mathrm{H}$ : Epigenetic alterations in cholangiocarcinomasustained IL-6/STAT3 signaling in cholangio-carcinoma due to SOCS3 epigenetic silencing. Digestion 79 (Suppl 1): 2-8, 2009.

61. Gao SM, Chen CQ, Wang LY, et al: Histone deacetylases inhibitor sodium butyrate inhibits JAK2/STAT signaling through upregulation of SOCS1 and SOCS3 mediated by HDAC8 inhibition in myeloproliferative neoplasms. Exp Hematol 41: 261-270, 2013. 
62. Liu SH, Wang KB, Lan KH, et al: Calpain/SHP-1 interaction by honokiol dampening peritoneal dissemination of gastric cancer in nu/nu mice. PloS One 7: e43711, 2012.

63. Xiong H, Du W, Zhang YJ, et al: Trichostatin A, a histone deacetylase inhibitor, suppresses JAK2/STAT3 signaling via inducing the promoter-associated histone acetylation of SOCS1 and SOCS3 in human colorectal cancer cells. Mol Carcinog 51: 174-184, 2012.

64. Shuai K and Liu B: Regulation of gene-activation pathways by PIAS proteins in the immune system. Nat Rev Immunol 5: 593-605, 2005.

65. Chung CD, Liao J, Liu B, et al: Specific inhibition of Stat3 signal transduction by PIAS3. Science 278: 1803-1805, 1997.

66. Saydmohammed M, Joseph D and Syed V: Curcumin suppresses constitutive activation of STAT-3 by up-regulating protein inhibitor of activated STAT-3 (PIAS-3) in ovarian and endometrial cancer cells. J Cell Biochem 110: 447-456, 2010.

67. Brantley EC, Nabors LB, Gillespie GY, et al: Loss of protein inhibitors of activated STAT-3 expression in glioblastoma multiforme tumors: implications for STAT-3 activation and gene expression. Clin Cancer Res 14: 4694-4704, 2008.

68. Redell MS, Ruiz MJ, Alonzo TA, Gerbing RB and Tweardy DJ: Stat3 signaling in acute myeloid leukemia: ligand-dependent and -independent activation and induction of apoptosis by a novel small-molecule Stat3 inhibitor. Blood 117: 5701-5709, 2011.

69. Fletcher S, Drewry JA, Shahani VM, Page BD and Gunning PT: Molecular disruption of oncogenic signal transducer and activator of transcription 3 (STAT3) protein. Biochem Cell Biol 87: 825-833, 2009.

70. Bromberg $J$ and Wang TC: Inflammation and cancer: IL-6 and STAT3 complete the link. Cancer Cell 15: 79-80, 2009.

71. Zhuang Q, Hong F, Shen A, et al: Pien Tze Huang inhibits tumor cell proliferation and promotes apoptosis via suppressing the STAT3 pathway in a colorectal cancer mouse model. Int J Oncol 40: 1569-1574, 2012.

72. Cai Q, Lin J, Wei L, et al: Hedyotis diffusa Willd inhibits colorectal cancer growth in vivo via inhibition of STAT3 signaling pathway. Int J Mol Sci 13: 6117-6128, 2012.

73. Lin W, Zheng L, Zhuang Q, et al: Spica prunellae promotes cancer cell apoptosis, inhibits cell proliferation and tumor angiogenesis in a mouse model of colorectal cancer via suppression of stat3 pathway. BMC Complement Altern Med 13: 144, 2013.

74. Kim SW, Park KC, Jeon SM, et al: Abrogation of galectin-4 expression promotes tumorigenesis in colorectal cancer. Cell Oncol 36: 169-178, 2013.

75. Kuno T, Hatano Y, Tomita $\mathrm{H}$, et al: Organo-magnesium suppresses inflammation-associated colon carcinogenesis in male Crj: CD-1 mice. Carcinogenesis 34: 361-369, 2013.

76. Uddin S, Hussain AR, Khan OS and Al-Kuraya KS: Role of dysregulated expression of leptin and leptin receptors in colorectal carcinogenesis. Tumour Biol: Sep 7, 2013 (Epub ahead of print).
77. Ganji PN, Park W, Wen J, et al: Antiangiogenic effects of ganetespib in colorectal cancer mediated through inhibition of HIF-1alpha and STAT-3. Angiogenesis 16: 903-917, 2013.

78. Zhu Z, Li E, Liu Y, et al: Inhibition of Jak-STAT3 pathway enhances bufalin-induced apoptosis in colon cancer SW620 cells. World J Surg Oncol 10: 228, 2012.

79. Huang S, Chen M, Ding X and Zou X: Proton pump inhibitor selectively suppresses proliferation and restores the chemosensitivity of gastric cancer cells by inhibiting STAT3 signaling pathway. Int Immunopharmacol 17: 585-592 2013.

80. Lamy S, Akla N, Ouanouki A, Lord-Dufour S and Beliveau R: Diet-derived polyphenols inhibit angiogenesis by modulating the interleukin-6/STAT3 pathway. Exp Cell Res 318: 1586-1596, 2012.

81. Chen JS, Chen YH, Huang PH, et al: Ginkgo biloba extract reduces high-glucose-induced endothelial adhesion by inhibiting the redox-dependent interleukin-6 pathways. Cardiovas Diabetol 11: 49, 2012.

82. Hedvat M, Huszar D, Herrmann A, et al: The JAK2 inhibitor AZD1480 potently blocks Stat 3 signaling and oncogenesis in solid tumors. Cancer Cell 16: 487-497, 2009.

83. Geletu M, Arulanandam R, Chevalier S, et al: Classical cadherins control survival through the gp130/Stat3 axis. Biochim Biophys Acta 1833: 1947-1959, 2013

84. Chang Y, Wang SX, Wang YB, et al: ECHS1 interacts with STAT3 and negatively regulates STAT3 signaling. FEBS Lett 587: 607-613, 2013

85. Ivanov VN, Wen G and Hei TK: Sodium arsenite exposure inhibits AKT and Stat3 activation, suppresses self-renewal and induces apoptotic death of embryonic stem cells. Apoptosis 18: 188-200, 2013.

86. Liu DB, Hu GY, Long GX, Qiu H, Mei Q and Hu GQ: Celecoxib induces apoptosis and cell-cycle arrest in nasopharyngeal carcinoma cell lines via inhibition of STAT3 phosphorylation. Acta Pharmacol Sin 33: 682-690, 2012.

87. Kang R, Tang D, Lotze MT and Zeh HJ III: AGER/RAGEmediated autophagy promotes pancreatic tumorigenesis and bioenergetics through the IL6-pSTAT3 pathway. Autophagy 8: 989-991, 2012.

88. Wu CT, Chen MF, Chen WC and Hsieh CC: The role of IL-6 in the radiation response of prostate cancer. Radiat Oncol 8: 159, 2013.

89. Yao X, Li G, Xu H and Lu C: Inhibition of the JAK-STAT3 signaling pathway by ganoderic acid A enhances chemosensitivity of HepG2 cells to cisplatin. Planta Med 78: 1740-1748, 2012.

90. Putoczki TL, Thiem S, Loving A, et al: Interleukin-11 is the dominant IL-6 family cytokine during gastrointestinal tumorigenesis and can be targeted therapeutically. Cancer Cell 24: 257-271, 2013. 